\title{
A Clockwork Orange: Compensation opposing momentum in memory for location
}

\author{
STEVE JOORDENS \\ University of Toronto at Scarborough, Toronto, Ontario, Canada \\ THOMAS M. SPALEK \\ Simon Fraser University, Burnaby, British Columbia, Canada \\ SAMIRA RAZMY \\ University of Toronto at Scarborough, Toronto, Ontario, Canada \\ and \\ MARC VAN DUIJN \\ Leiden University, Leiden, The Netherlands
}

\begin{abstract}
Libet, Gleason, Wright, and Pearl's (1983; Libet, 1985) influential work using a clock-watching task suggests that voluntary actions are initiated in motor cortex prior to the point where the participant claims to have initiated that action. Joordens, van Duijn, and Spalek (2002) showed that a bias exists in this task with respect to the participants' reports of initiation times. Joordens et al. assumed that this bias was primarily due to motion cues that are very much like those used to elicit phenomena such as representational momentum. In the present Experiment 1, it is demonstrated that this bias disappears when a mouse-click response is used in place of a temporal-order judgment. This finding, however, is actually more confusing than clarifying given that the procedural parallels with representational momentum are still present and should be supporting a bias. In the three subsequent experiments the view that a bias is indeed present, but that it is opposed by an opposite-acting compensation process, is proposed and tested. Implications for both representational momentum and for the general use of clockwatching tasks (e.g., Libet et al., 1983) are highlighted.
\end{abstract}

Although we often assume that memory provides us with an accurate record of past perceptual events, it is now apparent from a variety of studies that this is not always the case (e.g., Loftus, 1974). One factor that can cause distortions of memory is movement. In fact, movement can give rise to a number of memory errors including the Fröhlich effect (Fröhlich, 1923), the flash lag effect (Hazelhoff \& Wiersma, 1924; Nijhawan, 1994), and a phenomenon termed representationalmomentum (Freyd \& Finke, 1984). In the representational momentum phenomenon, if an object is moving as an observer watches it, that observer will be biased to remember its final location as being further along the trajectory of movement than it actually was. Thus, motion during encoding affects the way people remember an event (interested readers are directed to Thornton \& Hubbard, 2002, and to the 2002 special issue of Visual Cognition more generally).

It is the representational momentum phenomenon that forms the focus of the present work. In Freyd and Finke's (1984) original experiments, participants were presented with a series of three rotating rectangles. Following a 250-

Correspondence should be addressed to S. Joordens, Division of Life Sciences, University of Toronto at Scarborough, Scarborough, ON, M1C 1A4 Canada (e-mail: joordens@psych.utoronto.ca). msec blank interval, they were shown a probe item and asked to indicate whether it matched the final rectangle in the series in terms of orientation. Participants were more likely to false alarm to rectangles rotated further along the trajectory of rotation than to ones rotated less than the actual final rectangle.

Hubbard and Bharucha (1988) demonstrated that a similar error also occurs using slightly different procedures. Specifically, their display consisted of a dot that moved smoothly along either a vertical or horizontal trajectory. At some random point during the motion, the dot would disappear and participants were instructed to use the mouse to position the cursor over the final location of the dot and then to press the mouse button. Once again, the errors tended to be made further along the trajectory of motion.

One issue we wish to highlightis that such memory errors should be kept in mind anytime procedures are used that might support them (see Gray \& Thornton, 2001, for a similar argument). Consider the experiments by Libet, Gleason, Wright, and Pearl (1983; Libet, 1985). In these experiments, participants sat in front of a clock with electrodes measuring cortical activity in their motor cortex. The participants were instructed to wait for a while, then note the location of the hand on the clock and lift their arm. After performing this action, they were to report the noted location of the clock hand to the experimenter. The critical 
finding was that readiness potentials were observed in motor cortex prior to the reported initiation time. This finding was used to argue that perhaps humans do not will actions to occur but, rather, consciousness might simply be informed of an upcoming action. As long as the information is in consciousness prior to the act itself, an illusion of will could result (cf. Wegner, 2002).

Note that the procedure used by Libet et al. (1983) requires participants to watch a moving object, then to later report on its location-procedures that bear a striking similarity to those used in representational momentum studies. For example, an experiment by Müsseler, Stork, and Kerzel (2002, Experiment 2) involved the following procedures. A dot moved in a clockwise direction along a circular path and eventually disappeared. Participants were then asked to indicate both the original location of the moving dot and its final location. In this experiment, a reliable representational momentum effect was obtained with participants remembering the final location of the dot as being approximately $5 \mathrm{~mm}$ further along the trajectory of motion than it actually was.

Given the similarity of the procedures used by Libet et al. (1983) to those used by Müsseler et al. (2002), it seems likely that the reported initiation times provided by the participants in the Libet et al. study may also have been biased in a direction that would lead to later reported initiation times. If this is the case, then it is possible that the true initiation times would have been prior to the activity in motor cortex, thereby making the results more in line with intuitions about the causal nature of conscious intent.

In a 2002 special issue of Consciousness \& Cognition devoted to Libet's work, Joordens et al. (2002) tested the possibility that some form of bias might be affecting responses in clock-watching tasks. Specifically, one of the target articles in that special issue was a study by Trevena and Miller (2002) that replicated and extended the findings of the original Libet et al. (1983) experiments. ${ }^{1}$ Importantly, the procedures used by Trevena and Miller were slightly different from those used by Libet et al. In Trevena and Miller's experiment, participants again watched a target dot that moved around the perimeter of a vertically elongated rectangular clockface. At a point of the participants' choosing, they were to note where the target dot was and then initiate a buttonpress with either the left or right hand as indicated by an earlier cue. Immediately after making their buttonpress, a referent dot appeared somewhere along the perimeter of the clockface and participants were asked to perform a temporal-order judgment. This temporalorder judgment was accomplished by indicating whether the location of the target dot at the time of response initiation was before or after the location of the referent dot. ${ }^{2}$ Note that with these procedures, the participant are asked to localize where the dot was when a subjective event occurred (i.e., an internal decision to initiate a response) and, as such, they do not permit one to truly test the accuracy of those localizations.

To test whether any biases might be occurring in the reporting of the target dot's location, Joordens et al. (2002) modified the procedures used by Trevena and Miller(2002) in such a way that the event associated with localization was an objective event. Once again, participants were asked to watch a target dot move along the perimeter of a clockface that matched the one used by Trevena and Miller. At some point during this motion, the perimeter of the clockface changed color, and participants were asked to note the location of the target dot when that event occurred. The participants were also required to press a key to indicate they had noted the color change. A referent dot appeared and, as in the procedures of Trevena and Miller, participants were asked to make a temporal-order judgment by indicating whether the last location of the target dot was prior to or after the location marked by the referent dot. Because the target dot was at a known location at the time of the color change (i.e., this was an objectively measurable external event), it is possible to assess the accuracy of the participants in noting that location. A bias was indeed apparent, with participants indicating a dot location that was actually associated with a point $70 \mathrm{msec}$ further along the direction of movement than the actual location of the target dot when the clockface changed color.

Joordens et al. (2002) argued for two potential causes of the observed bias. First, as highlighted in this introduction, the procedures used in Trevena and Miller's (2002) study were very similar to those used to demonstrate representational momentum. The main procedural difference was that the dot continued moving beyond the "to-beremembered" point. However, the procedure was otherwise similar, and clearly some sort of bias was present. Thus, a momentum-like effect occurring in the context of the clock-watching task could cause the observed bias. In fact, Joordens et al. assumed this was the most likely explanation of their findings.

The second possibility is that the decision dynamics of the temporal-order judgment may have also supported a bias. This is actually a very complex issue that is not a focus of the present paper. Readers who are interested in a fuller discussion of this issue are directed to Joordens et al. (2002) and to Miller and Trevena (2002). However, the basic notion is this. In the Joordens et al. and Miller and Trevena studies, the proportion of actual before versus after trials was not balanced. In both studies, the referent dot occurred more often in a trajectory location that was after, rather than before, the actual target location of the dot. This resulted in more trials where the before response would have been the correct response. If under conditionsof uncertainty the participants were more likely to attempt to even out the number of before and after responses, they would likely demonstrate an apparent bias toward inaccurate after responses.

As suggested, this is all quite complicated. The important point for present purposes is that the temporal-order judgments used previously may not be optimal because of the possibility that biases may be inherent in the measure. However, if no probe was actually presented, then this bias should be eliminated. Therefore, a more appropriate localization measure, and one that is more similar to Libet's 
(1985) original task, might be to allow participants to point to the remembered location, for example by using a mouse-click response.

It is relevant to note that representational momentum has been observed using both mouse-click responses and judgments of an object relative to a probe (e.g., Hubbard, 1990). The fact that representational momentum effects can be observed using either mode of responding does not, however, mean that the momentum-like effect Joordens et al. (2002) observed was due to representational momentum. Perhaps the observed bias was due entirely to decision dynamics that were the result of the biased probe presentation. If this is true, there should be no bias left when a mouseclick procedure is used, and this is the empirical issue addressed in Experiment 1.

\section{EXPERIMENT 1 \\ A Mouse-Click Version of the Clock-Watching Task}

The purpose of this experiment was to assess the role that the temporal-order judgment played with respect to the bias in the clock-watching task as reported by Joordens et al. (2002). Our strategy was straightforward. The experiment was an exact replication of Joordens et al. with one simple change. Instead of asking participants to localize the target dot by indicating whether it occurred before or after a referent dot, they were simply asked to mouse-click on the location of the clockface where the target dot was when the clockface changed color. This allows a direct assessment of whether there is any bias to locate the target dot either before or after the actual location. In addition, given that the mouse-pointing task is more similar to the actual pointing task used by Libet et al. (1983), the present experiment provides a better examination of the role bias may have had in determining the results of those original experiments.

\section{Method}

Participants. The participants in this experiment were 10 undergraduate students from the University of Toronto at Scarborough. All participants had normal or corrected-to-normal vision. The participants were given $\$ 10$ Canadian in exchange for their assistance.

Procedure. Our procedures were a modified version of those used by Joordens et al. (2002) that allowed the use of a mouse click in place of the temporal-order judgment. Specifically, there were 12 blocks of 20 trials each. Each trial consisted of the following sequence of events: (1) the letter $\mathrm{L}$ or $\mathrm{R}$ was presented ${ }^{3}$ at the vertical and horizontal center of the screen for $1 \mathrm{sec}$; (2) a blank screen was presented for a duration randomly chosen from a continuous uniform distribution ranging from 1 to $2 \mathrm{sec}$; (3) a blue clock was presented; (4) a white dot appeared at a randomly chosen location on the clock's perimeter and began moving in a clockwise direction at a rate of 1 revolution every $406.25 \mathrm{msec}$; (5) after a randomly determined time sampled from a continuous uniform distribution ranging from 2 to $8 \mathrm{sec}$, the clock perimeter changed color from blue to yellow; (6) the dot continued to move while the program waited for the participants to hit either the "z" (L response) or "/" (R response) key to indicate they had noted the color change; (7) the dot continued to move for a duration randomly sampled from a continuous uniform distribution ranging from 500 to $800 \mathrm{msec}$; (8) a mouse pointer ap- peared at the middle of the clockface and the program paused until the participants moved the pointer to the location on the perimeter, where they estimated the dot to be when the clockface changed color, then clicked with the left mouse button; and (9) a blank screen was presented for $500 \mathrm{msec}$ and the next trial began automatically.

Participants were instructed to fixate the middle of the clock and to watch the dot move with their attention. The movement of the dot appeared as continuous motion, not as discreet jumps of the dot. In fact, the subjective impression was one of a dot moving quickly and smoothly along the perimeter of the clock, the clock perimeter then changing color, and the dot then continuing to move along its trajectory for a little while after the keypress was made.

A series of $4 \times 4$ pixel bins were located around the perimeter of the clock, and the mouse would only accept clicks that fell into one of these targets. For each click that was made, the distance between the bin location of the click and the target location of the dot when the clock perimeter turned color was calculated. In fact, given that the perimeter of the clock was continuous, two such distances were calculated, one measuring how far "behind" the actual dot location the selected bin was and another measuring how far "ahead" of the actual dot location the selected bin was. It was then assumed that the lesser of these two distances was the best reflection of the error (or bias) in locating the dot location. For the remainder of this paper, the average of these lesser distances for each participant will be termed the mean deviation score.

To ensure that the assumptions underlying the measurement of this mean deviation score are clear, consider the following analogy. A runner is on a continuous oval-shaped track that contains an explicit "start" line. If the runner is traveling at his typical speed, he completes one lap of a 200-m track in $1 \mathrm{~min}$. The question of interest is whether he is running faster or slower than average on this day. In order to assess this, one could allow him to run for 1 min and then see where he is relative to the start line (i.e., the target location). However, wherever he is on the track, his present position (i.e., the selected bin) is simultaneously in front of and behind the start line. That is, if he is currently at the $50-\mathrm{m}$ mark, then he is $50 \mathrm{~m}$ in front of the start line (i.e., +50 ), as well as $150 \mathrm{~m}$ behind the start line (i.e., -150 ). If he is less in front of the line than behind it, chances are he is running slightly faster than average and has crossed the line prior to the 1-min mark. In contrast, if he is less behind the line than in front of it, he is likely running slower than average and has not yet hit the line to begin his second lap.

Similarly, any click on the clock perimeter is simultaneously in front of and behind the actual dot location at the time of the color change. In total, there were 200 potential response bins located around the face of the clock. If the actual target location was assigned a deviation score of 0 , then the deviation scores from the actual target location could range from -199 bins (behind the target location) to +199 bins (in front of the target location). Because we took the lesser distance from the actual location as our measure of the deviation score, all of the measured deviations in Experiment 1 fell between -80 bins and +80 bins, with the majority being fewer than 20 bins from the target location (i.e., the 80-bin deviations were low-probability extreme scores). Given this, it seemed appropriate to use the lesser deviation scores as the measure of localization errors.

At this point it is relevant to note two aspects of our clock-watching task that differ from typical representational momentum procedures. First, as previously mentioned, the dot continued moving after the point where the clock perimeter changed color. Thus, participants are actually being asked to remember a location that is part way along a trajectory of motion, not a location associated with the final location of the dot. This difference from standard procedures will be addressed in Experiment 3. Second, the clock perimeter used here contained markings as indicated in Figure 1. Gray and Thornton (2001) found that the presence of markings can result in a diminished representational momentum effect. Given that momentum effects 


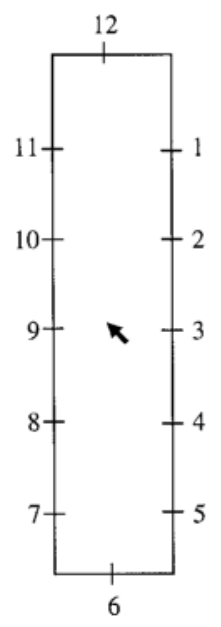

Figure 1. An illustration of the display as it would appear immediately after participants had pressed a key to indicate noticing the color change, but before they had moved the mouse. The arrow in the middle of the clock is the mouse cursor.

were observed in subsequent experiments of this paper, it appears that the markings did not result in the elimination of momentum biases, although it may have reduced their magnitude somewhat.

Stimuli and Apparatus. Figure 1 provides an illustration of the clock stimulus used in the present experiment as it would appear immediately after participants have indicated that they had noted a color change. The clock perimeter measured $15 \mathrm{~mm}$ wide and $110 \mathrm{~mm}$ high. The clock contained the numbers 1 through 12 arranged around its perimeter at equal intervals and with associated tick marks. The target dot that revolved around the clock perimeter was white and measured $1 \times 1 \mathrm{~mm}$. It appeared at a random location on the perimeter and traversed it at least once before the clock perimeter changed color. When present, the target dot occluded the perimeter of the clock and extended slightly beyond it. The dot did not completely occlude the tick marks associated with the numbers. The mouse pointer that appeared at the center of the clock was white, measured approximately $2 \times 2 \mathrm{~mm}$, and, as is typical of mouse pointers, was arrow shaped, pointing upward and slightly left.

The stimuli were presented on a 486, IBM-compatible computer attached to an SVGA color monitor. All testing was conducted in a lamp-lit room with a white-noise generator used to mask as much peripheral noise as possible. Responses were collected using the " $\mathrm{z}$ " and " " keys of the keyboard, and using an attached serial mouse.

\section{Results and Discussion}

Across the 10 participants tested in Experiment 1, there was an average bias of -3.02 bins (i.e., the reported location of the dot was at a location approximately $6 \mathrm{msec}$ before the color changed). This average was not significantly different from zero $[t(9)=1.40, S E=2.16]$. Thus, the tendency of participants to indicate the dot's location at the point of color change as occurring later along the trajectory of motion disappears when the temporal-order judgment is replaced by a mouse click. If anything, participants now indicate it at a location that is slightly, but not significantly, earlier along the trajectory of motion.
One interpretation of the present findings is that the bias in the clock-watching task reported by Joordens et al. (2002) was completely due to response biases inherent in the temporal-order judgment task. When this task is replaced by a mouse-pointing response, no systematic bias is observed. By this account, the mouse-pointing version of the clock-watching task is viewed as providing a bias-free timing task.

This lack of bias conclusion, however, is not completely satisfying. Given the striking similarities of the procedures used in Experiment 1 to those used in representational momentum papers (e.g., Müsseler et al., 2002), it is not at all clear why a momentum-like bias was not observed in our experiment. That is, the use of a mouse-click response in place of the temporal-order judgment did not alter the features of this task that make it similar to the typical representational momentum paradigm. So why was no representational momentum effect observed?

While considering this mystery, we must note that when participants are attempting to specify previous locations of an object in motion, performance may not be due to a single factor. Instead, a number of factors may work jointly to determine final performance. For example, in addition to the effects of implied momentum, representational momentum effects have also been shown to be sensitive to manipulations of implied gravity (Hubbard, 1990, 1995), implied friction (Hubbard, 1995; Hubbard \& Bharucha, 1988), memory averaging (Freyd \& Johnson, 1987), landmark attraction effects (Hubbard \& Ruppel, 1999), cognitive resistance (Finke, Freyd, \& Shyi, 1986), and reference frame effects (Gray \& Thornton, 2001). While some of these factors might promote errors, such as momentum and gravity effects, others may attenuate errors, such as friction effects, landmark effects, cognitive resistance, or reference frame effects. Thus, the resulting pattern may reflect how these effects mix to influence performance.

In the subsequent experiments, another factor is considered that may influence responding in our experiment, that factor being compensation processes. In our postexperiment debriefings with participants from Experiment 1, some indicated that they noticed the continued motion of the dot after the color change and worried that this motion might bias them to overestimate the color-change location of the target dot. Essentially, they were saying that they worried about something like the representational momentum effect. Further, they also claimed that because of this they actually tried to compensate slightly and choose locations earlier along the trajectory than they otherwise might have. If these reports are taken at face value, then perhaps a representational momentum type bias was occurring but it was being strategically compensated for by the participants.

Note that to the extent that compensation is occurring, this reflects a qualitatively different type of factor relevant to the momentum effects. That is, while there have been previous arguments for factors that attenuate the momentum effect, such as the notions of cognitive resistance and friction mentioned earlier, these factors are merely assumed to reduce the amount of representational momentum that is observed. Our view of the compensation mechanism is that 
it does more than attenuate momentum effects, it directly opposes them. In addition, previous factors assumed to affect the size of an observed momentum effect have been assumed to reflect low-level, automatic processes. In contrast, we assume that compensation is due to a higher level, controlled process.

The notion of a controlled process (e.g., compensation) opposing the influence of an automatic process (e.g., representational momentum) is not new to cognitive theories. For example, Merikle, Joordens, and Stolz (1997) illustrated this in the area of perception with and without awareness using what is called an exclusion task. In these experiments five letter words such as SPICE were presented briefly prior to a pattern mask, and were then followed by word stems such as SPI_. Participants were instructed to add two letters to the stem to create any English word other than the briefly presented word.

The theoretical notion here is that this context may provide a situation were controlled goal-oriented processes may oppose automatic processes. That is, the brief presentation of SPICE should prime its lexical representation automatically, resulting in an increased likelihood of it coming to mind as a completion. However, given the instructions, when participants were aware of seeing SPICE, they should instigate controlled processes that would oppose this automatic influence and instead support some other completion (e.g., SPIKE, SPILL, SPINE, etc.). In accord with this notion, participants showed an above chance likelihood of using the presented items when it was presented very briefly, but as exposures were lengthened participants showed a below chance likelihood of using the item as their completion (for other examples of contexts where controlled processes are assumed to oppose automatic influences see Cheesman \& Merikle, 1986; Groeger, 1984; Jacoby, 1991; Joordens \& Merikle, 1992; Merikle \& Joordens, 1997).

Thus, another way of thinking about the results of Experiment 1 is that they also reflect a balance of controlled and automatic influences. The motion of the dot automatically supports a momentum bias that influences participants to estimate the final location as further along the trajectory of motion than it actually was. However, cues within the experimental context inform participants that such a bias may be present, and the participants' take steps to compensate for it by actually choosing a location that is slightly backwards from the trajectory of motion. If momentum and compensation are present in equal measures, the result can be an apparent lack of bias, or perhaps better stated, a successful compensation for bias.

However, it is clearly tenuous to assume that any lack of an effect is due to two countervailing forces in perfect balance. The purpose of the remaining experiments is to test the compensation notion by examining variables that should affect either the momentum or the compensation force separately. If these forces truly are acting in opposition, and if they are in balance in the context examined in Experiment 1, then manipulationsintended to shift the balance should lead to predictable results.
In fact there is already one finding in the literature consistent with the compensation notion, although it was not interpreted in this manner. Hayes and Freyd (2002) examined the effects of divisions of attention on the magnitude of the representational momentum effect. By our view, dividing participants' attention should reduce their ability to employ a strategic process such as the compensation process highlighted here (see Merikle \& Joordens, 1997). If compensation opposes the momentum effect, as argued, and the momentum effect is due to automatic processes, then the net effect of dividing attention should be a larger momentum effect. In fact, this is exactly what Hayes and Freyd observed.

As mentioned, Hayes and Freyd (2002) did not interpret this finding in terms of a compensation process. Rather, they argued that as participants view a moving object, they form internal representations of that object that includes the motion that the object is undergoing. In order to accurately report the last position of a moving object, the participant must halt the movement of the internal representation. Dividing attention is assumed to interfere with the halting of the internal representation and, given that it is this internal representation upon which participants rely when making their decisions, the difficulty in halting the internal representation results in larger representational momentum effects. The contrast between the compensation and noncompensation (i.e., internal halting) views on the effects of dividing attention will be discussed throughout this paper.

Before delving too deeply into theory however, there are a number of differences between the procedures used by Hayes and Freyd (2002) and those typical of the clockwatching task as described in Experiment 1. Thus, as a first step in testing our compensation notion, performance on the clock-watching task was contrasted across conditions of single versus dual tasks, analogous to the manipulation performed by Hayes and Freyd. If the lack of bias observed in Experiment 1 was due to momentum and compensation being in balance, then this null effect should replicate in the single-task condition, but a momentumlike effect should be observed in the dual-task condition. Such a finding would not only support the proposed momentum versus compensation framework, but it would also strengthen the connection between the clock-watching task and typical representational momentum procedures if both show an augmentation of the momentum effect in a dualtask context.

\section{EXPERIMENT 2 Single Versus Dual task}

In the present experiment participants performed a task based on that described in Experiment 1 with one main alteration. On one block of trials the participants performed the identical task as in Experiment 1 and, as such, this single-task condition should produce a replication of the finding of no systematic memory bias. On another block of trials the participants had to perform the task used in Experiment 1 while concurrently performing a secondary task. If dividing attention interferes with the ability to com- 
pensate as we suggest, then this dual-task condition should allow the momentum bias to be observed.

\section{Method}

Participants. Fourteen undergraduate students from the University of Toronto at Scarborough participated in the present experiment. All participants had normal or corrected-to-normal vision. In exchange for their participation, each participant was either paid $\$ 5$ Canadian or given course credit.

Procedure. The present procedure was based on that used in Experiment 1 with a few slight modifications. First, rather than using the left versus right hand responses used in Experiment 1, in the present experiment participants were simply asked to strike the "b" key when they noticed the clock perimeter change color. Second, instead of the clock perimeter changing from blue to yellow, in this and subsequent experiments it changed from blue to orange. ${ }^{4}$ Finally, in the present experiment there were 24 blocks of 8 trials each; 12 blocks constituting the single-task context, and the other 12 constituting the dual-task context. Half of the participants performed the single-task condition first, and the other half completed the dualtask condition first.

In the dual-task condition participants performed the primary task as described in Experiment 1 while simultaneously listening via headphones to an auditory recording of a continuous stream of individually presented digits. They were instructed to monitor this recording for the occurrence of three odd digits in succession, and to mentally keep track of the number of times that such runs had occurred. Given the combined demands of monitoring the stream of digits and holding a memory load (the present count of how many odd digit triads had previously occurred), while also doing the clockwatching task, it is not surprising that the participants found this condition extremely demanding. However, all of the participants were able to successfully complete these trials. It should also be noted that the presentations of the three odd-digit runs were not linked to the visual presentation of stimuli in any systematic way.

Stimuli and Apparatus. The visual stimuli were presented on a 486, IBM-compatible computer attached to a SVGA color monitor. All testing was conducted in a lamp-lit room with a white-noise generator used to mask as much peripheral noise as possible. Given that the white noise generated was external to the apparatus (i.e., it is essentially a smoothly running ceiling fan), it played no role when participants were using the headphones. Responses were collected using the "b" key of the keyboard and an attached serial mouse.

The auditory task was a digit-monitoring task modeled after those used by Craik (1982), Jacoby (1991), and Merikle and Joordens (1997). The initial recording consisted of a stream of 1,051 digits read by a female voice with each digit being a number between 1 and 9. This auditory stream was presented to the individuals by way of headphones attached to an MP3 player. The MP3 player was a RIO 600 series. The digits were presented at a rate of 1 digit per second. The MP3 player allowed us to loop the recording with virtually no break in the stream, thereby allowing it to continue indefinitely. A digital copy of this recording is available from the "psychological stimuli" link at www.psychexperiments.com.

\section{Results and Discussion}

As in Experiment 1, the dependent measure of interest was the mean deviation score. Although participants were asked to note their running count for the digit-monitoring task, it is difficult to know for certain that all participants performed the task as instructed. All did provide estimates, though, that were within $2 S D$ of the correct value. Moreover, to the extent that any participant did not engage in the task as instructed, that would work against finding differential effects across the single- versus dual-task conditions.
Given that order of the single- versus dual-task conditions were counterbalanced in this and all subsequent experiments, the data were initially analyzed using an analysis of variance with order and condition as factors. However, order produced no significant main effects or interactions in any of these experiments (all $F_{\mathrm{S}}<2.6$, all $p \mathrm{~s}>.12$ ). Thus, to allow a clearer and more efficient presentation of the data, the order factor was collapsed across, and only $t$ tests examining condition effects are presented.

For the single-task condition, the mean deviation score was +1.47 bins (i.e., the indicated location of the dot was approximately $3 \mathrm{msec}$ after the actual color change). This value was not significantly different from zero $[t(13)=1.76$, $S E=0.84]$. Thus, replicating Experiment 1, no systematic bias was observed in a single-task context. In contrast, for the dual-task condition, the mean deviation score was +5.87 bins (i.e., the indicated location of the dot was approximately $12 \mathrm{msec}$ after the actual color change). This value was significantly different from zero $[t(13)=4.46, S E=1.32, p<$ $.001]$, suggesting that a momentum-like memory bias was observed. In addition, the deviation score for the dual-task condition was reliably larger than that observed in the singletask condition $[t(13)=4.72, S E=0.93, p<.001]$.

These findings are in accord with the predictions of the momentum versus compensation framework outlined in the discussion of Experiment 1. On the basis of the assumption that the compensation effect was the result of controlled processing, it was predicted that the division of attention would result in this compensation being decreased in the dual-task condition. This reduction of the influence of compensation processes should then allow a momentumlike bias to be observed. Consistent with this prediction a momentum-like bias was observed in the dual-task condition. Given this, the momentum effect is also assumed to be present in the single-task condition, but is being masked by an opposite-acting tendency to compensate on the part of our participants.

It is important to again note, however, that our interpretation of these findings is not the only one possible. Hayes and Freyd (2002) argued that divisions of attention, as are assumed to occur in dual-task contexts, augment the momentum effect by interfering with the halting of movement in the internal representation of the event. According to this view, the difficulty in halting the internal dynamics associated with the item would result in larger momentum effects, as were observed in the present experiment. Thus, while the present findings and those of Hayes and Freyd are consistent with our compensation view, they are also consistent with their noncompensation view.

\section{EXPERIMENT 3 Stopping the Dot}

Given the possibility of alternate explanations for results such as those described in Experiment 2, the purpose of Experiment 3 was to examine other factors, in addition to the dual-task context, that might affect the tendency of participants to compensate. In our attempts to identify such 
factors, the following question became relevant. If our assumption is correct that the procedures used to elicit momentum effects can also elicit compensation processes, then why are these compensation processes not eliminating momentum effects in the more conventional representationalmomentum paradigms? Of course, it may be the case that some compensation is occurring, but that the compensation is not sufficient to counteract the momentum effect. However, that still leaves the question open as to why compensation effects might be stronger in the clock-watching task than using more conventional representational momentum procedures.

There is one difference in procedure that may be important to the compensation processes. Specifically, as highlighted earlier, in the clock-watching task the dot continues to move after the critical event has occurred. However, in conventional representational-momentum paradigms, the stimulus disappears at the to-be-remembered location. Perhaps the continued motion of the dot helps to trigger (or strengthen) the compensation processes and that is why they are stronger in the context of the clock-watching task than in more conventional representational momentum tasks. Said another way, the tendency of the dot to continue moving may provide a strong cue to the participant concerning the potential bias that motion might support and, as such, may serve to trigger or strengthen the desire to compensate.

If this is the case, then if the procedures of Experiment 2 were altered such that the dot did not continue to move to the same extent, then the tendency to compensate should be generally reduced, perhaps permitting momentum like effects to be observed in both the single-task and dual-task conditions. If a reduction in the continued motion completely eliminated compensation processes then, by our account, similar momentum biases should be observed in both the single- and dual-task contexts. However, if compensation is still present but to a lesser extent, then momentum effects should be larger in this than in the singletask conditions of the previous experiments, and there should still be a larger momentum effect in the dual-task context given that the tendency to compensate should be even further reduced in that context.

\section{Method}

Participants. Fourteen undergraduate students from the University of Toronto at Scarborough participated in the present experiment. All participants had normal or corrected-to-normal vision. In exchange for their participation, each participant was either paid \$5 Canadian or given course credit.

Procedure. The procedures of this experiment were identical to Experiment 2 except for one minor alteration. In this experiment, the target dot disappeared immediately when participants made their keypress to note the color change. Thus, the color of the clockface changed and as soon as participants indicated awareness of that change, the target dot disappeared. The result of this manipulation is that the target dot continued to move only for the duration of a simple detection response (i.e., approximately $200 \mathrm{msec}$ ) which is approximately 500 to $800 \mathrm{msec}$ less continual motion than occurred in Experiments 1 and 2.

Stimuli and Apparatus. The stimuli and apparatus were identical to those used in Experiment 2.

\section{Results and Discussion}

The mean deviation score for the single-task context was +5.54 bins (i.e., a location approximately $11 \mathrm{msec}$ further along the trajectory of movement). This mean was significantly different from zero $[t(13)=4.93, S E=1.12, p<$ $.001]$. Thus, when the dot did not continue moving after the keypress a significant momentum effect was observed even in the single-task condition. The mean deviation score in the dual-task condition was +8.52 bins (i.e., a location approximately $17 \mathrm{msec}$ further along the trajectory of movement). This mean was significantly different from both zero $[t(13)=5.36, S E=1.59, p<.10]$, and from the mean in the single-task condition $[t(13)=4.90, S E=1.12, p<$ $.010]$. Thus, a reliable momentum effect was also observed in the dual-task condition, and that effect was larger than the one that was observed in the single-task condition.

These findings suggest that the continued movement of the dot (as in Experiment 2) does indeed strengthen the tendency for participants to compensate. That is, when the continued movement was reduced, a momentum-like bias was observed in the single-task context, which was a context where it was not observed when the continued motion of the dot was more extreme (i.e., Experiments 1 and 2). However, the reduction in the continued motion appears not to have completely eliminated the tendency to compensate as an even bigger bias is observed when this manipulation is performed in a dual-task context. By our account this occurs because there is still some compensation occurring in the single-task context, and the dual-task task manipulation reduces that compensation further.

These results appear somewhat problematic for the noncompensation view of divided attention proposed by Hayes and Freyd (2002). Recall that Hayes and Freyd argue that divisions of attention produce stronger momentum effects via interference with processes that halt the movement of the internal representation. By this view one might also expect that continued motion of the dot after some critical event might also interfere with one's ability to halt an internal representation of that moving object. Thus, one might expect it to be easier to halt the internal representation when the dot stops. However, this would lead to the prediction of a smaller momentum effect with reduced dot motion beyond the color change, which was the opposite of what was observed in the present experiment. Of course, this prediction is derived from our perspective of their view, and it could be that they could provide a different explanation within their framework that would allow them to explain larger momentum effects when the dot continues moving to a lesser extent. At best, all that can be said is that the data from Experiment 3 require such an explanation.

\section{EXPERIMENT 4 Manipulating the Momentum Component}

In Experiments 2 and 3, the focus was on factors assumed to target the compensation component of our momentum versus compensation framework. However, the 
case for countervailing momentum versus compensation processes would be stronger if it could be demonstrated that a factor assumed to primarily affect the momentum component also gives rise to predicted effects. That was the goal of Experiment 4.

A number of studies have revealed that there are systematic asymmetries in the representational momentum effect. For example, momentum effects are larger when the stimulus is moving from left-to-right than when it is moving from right-to-left (Halpern \& Kelly, 1993). Momentum effects are also larger for objects moving in the direction of gravitational attraction (Hubbard, 1995; Nagai, Kazai, \& Yagi, 2002; see also Hubbard, 1990, and Nagai \& Yagi, 2001 , for examples of other asymmetries). Spalek and Hammad (2003) argued that such asymmetries likely reflect the effect of environmental learning. That is, things tend to move in certain ways in the environment, and our visual systems incorporate these tendencies in a way that affects the extent to which momentum-like effects will be observed (see also Hubbard, 1999).

By this logic, there should be relatively strong environmental tendencies in a clock-watching context. This is due to the fact that all analog clocks display clockwise motion, and as a result our visual (and memorial) systems have likely incorporated this regularity. As indicated in Figure 1, while our stimulus may not fit one's stereotype of a clock, it should be sufficiently clock-like in appearance to bring to mind a "clock" schema, thereby supporting an expectation for clockwise rotation. Given that this is the direction that is consistent with the natural tendency, momentum effects should be maximal in these situations. If, instead, the dot moved in a counterclockwise manner, we might expect less of a momentum effect. ${ }^{5}$ Thus, by reversing the direction of movement of the dot it should be possible to reduce the strength of the momentum component.

It is possible that, within our experimental context, participants would not be sensitive to the asymmetries in the representational momentum effect that may be associated with clockwise versus counter-clockwise directions of movement. If this is true then their tendency to compensate might not be affected by this manipulation. This leads to an intriguing possibility. If the tendency to compensate remained high, but the momentum effect were reduced, it might be possible to observe a systematic bias that runs counter to the typical momentum effect (i.e., an overcompensation). Such a negative-momentumeffect should only occur if there is, indeed, a force acting in opposition to the momentum effect as proposed.

\section{Method}

Participants. Sixteen undergraduate students from the University of Toronto at Scarborough participated in the present experiment. All participants had normal or corrected-to-normal vision. In exchange for their participation, each participant was either paid \$5 Canadian or given course credit.

Procedure. Given that the desire to keep the tendency to compensate high, the continued motion version of the clock-watching task used in Experiment 2 was also used here as it is assumed to produce the greatest tendency to compensate. In fact, the only change from the procedures of Experiment 2 was that, rather than the dot moving in a clockwise direction, it moved in a counter-clock wise direction.

Stimuli and Apparatus. The stimuli and apparatus in the present experiment were identical to those used in Experiment 2.

\section{Results and Discussion}

The mean deviation score for the single-task condition was -2.36 bins (i.e., the indicated location of the dot was approximately $5 \mathrm{msec}$ before the color change). This mean was significantly less than zero $[t(15)=2.16, S E=$ $1.09, p<.05]$. Thus, changing the direction of rotation in a context where participants are assumed to be compensating resulted in a reliable negative momentum effect. This is exactly what would be expected if participants were overcompensating for a reduced momentum effect.

The mean deviation score in the dual-task condition was +0.59 bins (i.e., the indicated location of the dot was approximately $1 \mathrm{msec}$ after the color change location). This mean is not significantly different from zero $[t(15)=0.33$, $S E=1.79]$. By our account, this nonsignificant result occurs because this is a context in which both the momentum effect and the tendency to compensate are reduced significantly due to the effects of the direction of motion and dual task respectively. That is, the negative momentum observed in the single-task context is assumed to be due to overcompensation, and if dividing attention by presenting the task in a dual-task context serves to reduce one's ability to compensate, it seems reasonable this overcompensation would not be observed in the dual-task condition. The deviation score for the single-task condition was also significantly less than the mean deviation score for the dualtask condition $[t(15)=2.47, S E=1.19, p<.03]$.

Thus, once again, the results fit nicely with the predictions of the momentum versus compensation framework. In fact, a complete reversal of the typical representational momentum was observed simply by sufficiently reducing momentum relative to compensation. Even if one does not like our assumptions concerning compensation, the finding of a reversed effect clearly shows that something is countering the momentum effects, and compensatory processes seem the most likely candidate at this point.

Returning one more time to the Hayes and Freyd (2002) interpretation of the effects of divided attention on representational momentum, one more point seems relevant at this stage. Not only do we see the results of Experiment 3 as problematic for their view as outlined in the discussion of that experiment, but we also wish to note that their account would not predict a reversal of the momentum effect in the present context. That is, given that they see divisions of attention as relevant to the same process that produces momentum effects - the need to halt the dynamics of the internal representation - anything that reduces momentum should simply result in smaller effects overall. There is no reason why the effect should ever be significantly reversed. A significant reversal implies a counteracting force to momentum, and their view includes no counteracting force. Thus, once again, we see our momentum versus compensation framework as providing the best overall fit to the data. 
Table 1

Summary of Both the Theoretical Assumptions and Associated Data Patterns From All the Empirical Conditions Examined in Experiments 1-4

\begin{tabular}{ccc}
\hline \multirow{2}{*}{ Compensation Processes } & \multicolumn{2}{c}{ Momentum Effect } \\
\cline { 2 - 3 } Weak & Weak & Strong \\
\hline \multirow{2}{*}{ Medium } & & Exp. 3-Dual \\
& & $+8.52^{*}$ \\
& & Exp. 3-Single \\
& & $+5.54^{*}$ \\
Strong & Exp. 4-Dual & Exp. 2-Dual \\
& +0.59 & $+5.87^{*}$ \\
& Exp. 4-Single & Exp. 2-Single \\
& $-2.36^{*}$ & +1.47 \\
& & Exp. 1-(Single) \\
& & -3.00 \\
\hline
\end{tabular}

$* p<.05$.

\section{GENERAL DISCUSSION}

The present paper represents a further attempt to understand the influences that may become relevant when one is asked to indicate a previous position of a moving object. Experiment 1 presented a mystery of sorts. By using a mouse click response instead of a temporal-order judgment the momentum-like bias in the clock-watching task originally noted by Joordens et al. (2002) was effectively eliminated. This finding is surprising because the mouse click version of the clock watching task utilizes procedures that run parallel to those typically used in experiments that have repeatedly shown a memory bias in the representational momentum literature. Why was no momentum-like bias observed in our task when it possesses all of the characteristics assumed to produce momentum effects?

In Experiments 2-4, the view that the procedures that give rise to momentum effects also give rise to counteracting compensation processes was proposed and tested. The assumption underlying these tests was that while the momentum effects reflect automatic processes, the compensation effects are the result of controlled processes initiated by the participants, and partly dependent on the experimental context. Specifically, continued motion of the stimulus in question strengthens the tendency to compensate, but dual-task conditions decrease the ability to compensate because the cognitive resources necessary to carry out the compensation must be shared with the secondary task. As in Hubbard (1990) and Halpern and Kelly (1993), momentum effects are also partly dependent on the experimental context, becoming larger in contexts where the motion is consistent with environmental tendencies.

A theoretical taxonomy of our findings is summarized in Table 1. We created this table in a post hoc manner by arranging the experimental conditions and their results to reflect our assumptions concerning the relative strength of the momentum and compensation effects relevant to those conditions. That is, Experiment 1 and the single-task condition of Experiment 2 are assumed to reflect conditions in which both the momentum bias and the tendency to compensate were strong. Relative to these conditions, the "stop motion" procedure used in Experiment 3 was assumed to reduce the tendency to compensate while leaving the momentum effect strong. Similarly, relative to Experiment 1 and the single-task condition of Experiment 2, the counterclockwise manipulation used in the single-task condition of Experiment 4 was assumed to weaken the momentum effect while not affecting the tendency to compensate. Once we had placed the single-task conditions in the table, we added the data from the dual-task conditions, assuming that this dual-task manipulation resulted in a one-level reduction of the compensation process on the reasoning that compensation is more difficult under dual-task conditions.

As illustrated, the resulting taxonomy provides a nice fit with the observed effects. The strongest momentum effect is observed in the context where momentum is assumed to be strongest, and the tendency to compensate the weakest. Staying in the "strong momentum" column but increasing the tendency to compensate, the momentum effect reduces in magnitude, eventually becoming nonsignificant when compensation is strong. In the "weak momentum" column, a medium tendency to compensate is sufficient to eliminate any reliable momentum effect, and a strong tendency to compensate actually results in a reversed momentum effect. Clearly the momentum versus compensation framework is supported by our data. The compensation versus momentum framework was arrived at by combining two research areas: studies on representational momentum and studies on the clock-watching task. Consistent with this genesis, implications of our work can be drawn with respect to both of these areas. Each will be discussed in turn.

\section{Representational Momentum}

Our framing of representational momentum in the present paper may have suggested a leaning toward a learning view of this phenomenon. While the learning view does provide a clear account of some of the asymmetries in the momentum effect, we also wish to acknowledge that there are alternate or more specific theories that have been applied to the basic representational momentum phenomenon (e.g., Finke et al., 1986; Freyd, 1987; Hubbard, 1998, 1999; Kerzel, 2000; Kerzel, Jordan, \& Müsseler, 2001; Kozhevnikov \& Hegarty, 2001). The factors highlighted in these theories may work instead of, or in addition to, the learning of environmental regularities to produce what we have termed the momentum effect. It was not our intention in the present work to discriminate among these views; to the extent that the learning view was favored over others, it was more for the sake of simplicity than it was to suggest the superiority of that view.

Our critical point, though, is that whatever may cause the momentum bias, there appears to also be a factor that opposes it. That is, while others have highlightedfactors that contribute to or attenuate the basic effect, to the best of our knowledge this paper is the first to highlight a process that may directly oppose it. Perhaps the closest notion in the literature is what Finke et al. (1986) termed cognitive resistance. However, cognitive resistance was used to refer to 
something more analogous to braking - that is, something that could be applied to reduce the amount of momentum. The compensation notion is more akin to reversing than to braking. That is, it is something that actively works against momentum to the extent that it can actually reverse the pattern completely, not just slow it down.

While the results of all the present experiments are consistent with the compensation versus momentum view, the strongest evidence comes from the reversed momentum effect observed in Experiment 4. How could a reliable reversed effect be observed unless some process were working in a direction that opposes the basic momentum effect? The cognitive resistance notion simply cannot account for this pattern. To us, compensation processes seem the best candidate for such an opposing process, and it is their existence that is highlighted here.

There is one finding in the literature that appears to not fit well with the compensation notion. Specifically, Finke and Freyd (1985) argued that representational momentum is not influenced by error feedback. Moreover, Freyd (1987) claimed that "the effect seems to be impervious to extensive knowledge of the predicted results and attempts to compensate for the effect" (p. 433). These findings appear to suggest that participants not only do not compensate in reaction to errors but perhaps also that they cannot compensate even when sufficient information is provided. If true, this would clearly show the compensation notion advanced here to be incorrect.

The original Finke and Freyd (1985) study is highly relevant with respect to determining the extent to which Freyd's (1987) "impervious to error feedback" claim is problematic for our compensation framework. The display used in Finke and Freyd's experiments involved three dots that were originally arranged as triangle and then, across three separate inducing trials, the dots each moved off in independent directions. Participants were then shown probes in which the dots were in their final location (the correct match to the final location) or either further along or less further along their respective paths of motion (the mismatching probes). Participants were given very general error feedback during the practice trials via a tone that sounded when errors were made. Despite this feedback, on the experimental trials the participants showed a tendency to false alarm more to mismatching probes further along the path of motion than to those less far along the path of motion (i.e., representational momentum). This finding was replicated across three experiments.

Two points are highly relevant with respect to Finke and Freyd's (1985) procedures. First, feedback was provided only in the 32 practice trials and was discontinuedonce the 128 experimental trials had begun. In the experimental trials, false alarms averaged about $30 \%$ and, assuming this is also true of practice trials as well, that means that of the 16 mismatching practice trials, participants would get feedback on about 5 trials. Second, the feedback that they did receive did not indicate the direction of the error to the participants, only that they were wrong. Thus, there was noth- ing to inform the participants that they were tending to choose mismatching probes further along the path of motion. Thus, although feedback was provided, it was done in a very information poor setting, and it is not overly surprising that it did not affect performance.

That said, we do agree that a strong demonstration showing the representational momentum is impervious to error feedback would be problematic for our claims. However, such a demonstration needs to provide more relevant information to the participants on a larger proportion of trials, and it should directly compare feedback with nofeedback conditions to truly assess what effect the presence of feedback has. Thus, while we see Freyd's (1987) claim as suggestive of the need for further investigations, we do not see these results as a major problem for our framework at this time.

Thus, in general terms the primary contribution of our work with respect to those interested in momentum biases is with respect to the possibility that momentum effects may be even larger than those typically reported. That is, depending on the experimental context, compensation processes may be working against the momentum effects of interest, making them appear smaller than they actually are. Fortunately, the present experiments suggest that dualtask contexts can be employed to reduce the tendency to compensate, thereby providing a purer measure of the true momentum effects that may be occurring.

\section{The Clock-Watching Task}

With respect to the clock-watching task, the news is not so good. Specifically, one reading of the Experiment 1 results suggests that the mouse-click version of the clockwatching task may be unbiased. However, the subsequent experiments suggest that the apparent lack of a bias likely reflects a subtle balancing act between momentum effects and compensation processes. It would be unwise to assume that this balancing act would remain stable across participant groups, experimental contexts, or both. Moreover, depending on how things were put out of balance, either of two biases would be possible depending on whether the momentum effect or the compensation process came to dominate. This all suggests that the reliability of the clockwatching task as an accurate timing measure is questionable at best.

That said, as one of our reviewers noted, the biases reported in the present paper are not overly large (i.e., 10$17 \mathrm{msec}$ ). Given present debates about how best to measure cortical activity (see Trevena \& Miller, 2002), it is difficult to say exactly how far cortical activity may precede a claim of subjective initiation of movement. It is unlikely, though, that the biases we report would account for all of this difference in time between cortical activation and subjective initiation of movement. Given this, perhaps some would consider the biases we report as reflecting an acceptable level of error in measurement, even if it is systematic in direction. Again, doing so would be tenuous since it is not clear how the various contrasting influences may 
play out within a given empirical context. At the very least, those using the clock-watching task to measure internal subjective events should include a condition with an objective external event that can be used to assess the bias in measurement associated with that specific context.

\section{Summary}

This paper provides data that fit with the notion that the automatic biases supporting momentum-like effects may sometimes be opposed by compensatory processes elicited by the experimental context. These findings are in line with the more general notion that in every experimental context performance is likely affected by a range of factors that sometimes work in concert and sometimes in opposition. Disentangling these factors may be a necessary first step to understanding the factors themselves.

\section{REFERENCES}

CheEsman, J., \& MERIKLE, P. M. (1986). Distinguishing conscious from unconscious processes. Canadian Journal of Psychology, 40, 343 367.

CRAIK, F. I. M. (1982). Selective changes in encoding as a function of reduced processing capacity. In F. Klix, J. Hoffman, \& E. van der Meer (Eds.), Cognitive research in psychology (pp. 152-161). Berlin: Deutscher Verlag der Wissenschaften.

FINKE, R. A., \& FREYD, J. J. (1985). Transformations of visual memory induced by implied motions of pattern elements. Journal of Experimental Psychology: Learning, Memory, \& Cognition, 11, 780-794.

FINKE, R. A., FrEYD, J. J., \& SHYI, G. C. W. (1986). Implied velocity and acceleration induce transformations of visual memory. Journal of Experimental Psychology: General, 115, 175-188.

FREYD, J. J. (1987). Dynamic mental representations. Psychological Review, 94, 427-438.

FREYD, J. J., \& FINKE, R. A. (1984). Representational momentum. Journal of Experimental Psychology: Learning, Memory, \& Cognition, 10, 126-132.

FREYD, J. J., \& Johnson, J. Q. (1987). Probing the time course of representational momentum. Journal of Experimental Psychology: Learning, Memory, \& Cognition, 13, 259-268.

FRÖHLICH, F. W. (1923). Über die Messung der Empfindungszeit [Measuring the time of sensation]. Zeitschrift für Sinnesphysiologie, 54, 5878.

Gray, R., \& Thornton, I. M. (2001). Exploring the link between time to collision and representational momentum. Perception, 30, $1007-$ 1022.

GROEGER, J. A. (1984). Evidence of unconscious semantic processing from a forced error situation. British Journal of Psychology, 75, 305314.

Halpern, A. R., \& Kelly, M. H. (1993). Memory biases in left versus right implied motion. Journal of Experimental Psychology: Learning, Memory, \& Cognition, 19, 471-484.

HAYES, A. E., \& FREYD J. J. (2002). Representational momentum when attention is divided. Visual Cognition, 9, 8-27.

HAZELhOFF, F. F., \& WIERSMA, H. (1924). Die Wahrnehmungszeit [The sensation of time]. Zeitschrift für Psychologie, 96, 171-188.

HuBBARD, T. L. (1990). Cognitive representations of linear motion: Possible direction and gravity effects in judged displacement. Memory \& Cognition, 18, 299-309.

HUBBARD, T. L. (1993). The effects of context on visual representational momentum. Memory \& Cognition, 21, 103-114.

HubBARD, T. L. (1995). Environmental invariants in the representation of motion: Implied dynamics and representational momentum, gravity, friction, and centripetal force. Psychonomic Bulletin \& Review, 2, 322-338.

HubBard, T. L. (1998). Representational momentum and other displacements in memory as evidence for nonconscious knowledge of physical principles. In S. Hameroff, A. Kaszniak, \& A. Scott (Eds.), Towards a science of consciousness: II. The second Tucson discussions and debates (pp. 505-512). Cambridge, MA: MIT Press.

HuBBARD, T. L. (1999). How consequences of physical principles influence mental representation: The environmental invariants hypothesis. In P. R. Killeen \& W. R. Uttal (Eds.), Fechner Day 99: The End of Twentieth-Century Psychophysics: Proceedings of the 15th annual meeting of the International Society for Psychophysics (pp. 274-279). Tempe, AZ: International Society for Psychophysics.

HubBaRd, T. L., \& BhaRUCHA J. J. (1988). Judged displacement in apparent vertical and horizontal motion. Perception \& Psychophysics, 44, 211-221.

Hubbard, T. L., \& RupPeL, S. E. (1999). Representational momentum and landmark attraction effects. Canadian Journal of Experimental Psychology, 27, 294-301.

JACOBY, L. L. (1991). A process dissociation framework: Separating automatic from intentional uses of memory. Journal of Memory \& Language, 30, 513-541.

JoORDENS, S., \& MERIKLE, P. M. (1992). False recognition and perception without awareness. Memory \& Cognition, 20, 151-159.

JoORdEnS, S., van DuiJn, M., \& SPALEK, T. M. (2002). When timing the mind one should also mind the timing: Biases in the measurement of voluntary actions. Consciousness \& Cognition, 11, 231-240.

KerZEL, D. (2000). Eye movements and visible persistence explain the mislocation of the final position of a moving target. Vision Research, 40, 3703-3715.

KERZEL, D., JoRDAN, J. S., \& MüsSELER, J. (2001). The role of perceptual anticipation in the mislocation of the final position of a moving target. Journal of Experimental Psychology: Human Perception \& Performance, 27, 829-840.

Kozhevnikov, M., \& Hegarty, M. (2001). Impetus beliefs as default heuristics: Dissociation between explicit versus implicit knowledge about motion. Psychonomic Bulletin \& Review, 8, 439-453.

Libet, B. (1985). Unconscious cerebral initiative and the role of conscious will in voluntary action. Behavioral \& Brain Sciences, 8, 529566.

Libet, B., Gleason C. A., Wright, E. W., \& Pearl, D. K. (1983). Time of conscious intention to act in relation to onset of cerebral activity (readiness-potential): The unconscious initiation of a freely voluntary act. Brain, 106, 623-642.

LOFTUS, E. (1974). Reconstructing memory: The incredible eyewitness. Psychology Today, 8, 116-119.

Merikle, P. M., \& JoORdens, S. (1997). Parallels between perception without attention and perception without awareness. Consciousness \& Cognition, 6, 219-236.

Merikle, P. M., Joordens, S., \& Stolz, J. A. (1997). Measuring the relative magnitude of unconscious influences. Consciousness \& Cognition, 4, 422-439.

Miller, J., \& TrevenA, J. A. (2002). Cortical movement preparation and conscious decisions: Averaging artifacts and timing biases. Consciousness \& Cognition, 11, 308-313.

Müsseler, J., StORK, S., \& Kerzel, D. (2002). Comparing mislocalizations with moving stimuli: The Fröhlich effect, the flash-lag, and representational momentum. Visual Cognition, 9, 120-138.

NAGAI, M., KAZAI, K., \& YAGI, A. (2002). Larger forward displacement in the direction of gravity. Visual Cognition, 9, 28-40.

NAGAI, M., \& YAGI, A. (2001). The pointedness effect on representational momentum. Memory \& Cognition, 29, 91-99.

NiJHAWAN, R. (1994). Motion extrapolation in catching. Nature, 370, 256-257.

SPALEK, T. M., \& HAMmAD, S. (2003). Supporting the attentional momentum view of inhibition of return: Is attention biased to go right? Manuscript submitted for publication.

Thornton, I. M., \& HubBARD, T. L. (2002). Representational momentum: New findings, new directions. Visual Cognition, 9, 1-7.

TrevenA, J. A., \& Miller, J. (2002). Cortical movement preparation before and after a conscious decision to move. Consciousness \& Cognition, 11, 162-190.

WeGner, D. M. (2002). The illusion of conscious will. Cambridge, MA: MIT Press. 


\section{NOTES}

1. Although Trevena and Miller (2002) did replicate the finding that generalized readiness potentials occur in motor cortex prior to the reported initiation time by participants, they pointed out that generalized readiness potentials can reflect things other than actual motor initiation. They also examined lateralized readiness potentials, which, they argued, provide better cortical indicators of motor initiation. These lateralized potentials were not observed to occur prior to the initiation point, as subjectively reported by participants. Thus, while Trevena and Miller replicated the basic Libet et al. (1983) pattern, they did not agree with Libet's contentions concerning the epiphenomenal nature of voluntary actions.

2. As correctly noted by one of our reviewers, it is somewhat misleading to call this task a temporal-order judgment. That is, participants are actually being asked "where" the dot was relative to a referent, as opposed to being asked "when" some event occurred relative to a referent. However, given that this was the terminology used in the described papers, and given we will not actually use this task in the present work, we felt it might be misleading and distracting to alter the terminology here.

3. The presentation of the $L$ versus $R$ is a legacy characteristic from the original replication of Trevena and Miller (2002). Because Trevena and Miller were interested in lateralized potentials, these cues informed participants which hand they were to respond with. Joordens et al. (2002) also had participants use their left or right hands in order to keep their replication as procedurally close to Trevena and Miller as possible. Given that the present experiments use mouse-click localizations, the $\mathrm{L}$ versus R distinction is moot. Nonetheless the display was retained in the present experiment to keep the procedures here identical to those used by Joordens et al. except for the change in localization response.

4. S.J. decided on this change partly as an excuse to use the term "Clockwork Orange" in the title. Aside from providing an accurate mnemonic for the empirical procedures used, the opposition of competing forces depicted in the movie with the same title captures the oppositional interaction between momentum and compensation proposed in the present work.

5. There have been previous failures to find an effect of clockwise versus counter-clockwise motion on the representational momentum effect (e.g., Hubbard, 1993), and this would seem to argue against our logic. However, while those studies utilized circular motion patterns, they did not include an explicit reference to a clockface, and it is this aspect of our procedure that may be critical for stimulating the environmental support for clockwise motion.

(Manuscript received October 10, 2002; revision accepted for publication August 16, 2003.) 